\title{
General Recursive Functions in the Number-Theoretic Formal System
}

\author{
By Shôji Maehara \\ Waseda University, Tokyo
}

The purpose of the present paper is to give a definition of general recursive functions formalized in the number-theoretic formal system and to show the calculability of those functions which we shall call simply 'recursive function' in this paper.

1. The number-theoretic formal system

As the number-theoretic formal system, in this paper, we use one formalized in Gentzen [2], which will be briefly explained in the following.

1. 1. 'Formula'

As individual and function symbols we use only 0 (zero) and '(successor function), respectively. Accordingly, by term we mean either one of the forms $0,0^{\prime}, 0^{\prime \prime}$ etc. - they are called numerals-or $\mathfrak{a}, \mathfrak{a}^{\prime}, \mathfrak{a}^{\prime \prime}$ etc., where $\mathfrak{a}$ is an arbitrary free variable. As predicate symbols we use = (equals) and any other predicate symbols which are not only decidable ones, as in Gentzen [2], but also undecidable ones, provided that they are defined for all non-negative integers. We use only formulae constructed by the ordinary formation rule of formula in the restricted predicate calculus from the above terms, those predicate symbols, the bound variables and the logical symbols : $>$ (not), \& (and), $\vee$ (or), $\supset$ (implies), $V$ (for all)

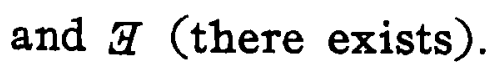

When $\operatorname{Vtq} \mathfrak{F}(\mathfrak{x})$ or $\mathbb{Z} \mathfrak{x} \mathfrak{F}(\mathfrak{x})$ is a formula of such form, $\mathfrak{F}(\mathfrak{t})$ means the result of substituting an arbitrary term $t$ for the bound variable $x$ 
throughout $\mathfrak{F}(\mathfrak{x})$.

We shall use Greek small letters to represent finite sequences of zero or more terms or bound variables, when we wish to indicate sets of terms or bound variables without naming them individualy. For example, if $\tau$ stands for a sequence of terms $t_{1}, t_{2}, \cdots \cdots, t_{n}$ and $\xi$ stands for a sequence of bound variables $\mathfrak{x}_{1}, \mathfrak{k}_{2}, \cdots \cdots, \mathfrak{x}_{n}$, then $\mathfrak{F}(\tau)$ means $\mathfrak{F}\left(\mathfrak{t}_{1}, \mathfrak{t}_{2}, \cdots\right.$ $\left.\cdots, \mathfrak{t}_{n}\right)$ and $\forall \xi \mathfrak{F}(\xi)$ means $V \mathfrak{x}_{1} \forall \mathfrak{k}_{2} \cdots \cdots \cdot \forall \mathfrak{x}_{n} \mathfrak{F}\left(\mathfrak{x}_{1}, \mathfrak{x}_{2}, \cdots \cdots, \mathfrak{x}_{n}\right)(n=0,1,2, \cdots \cdots)$.

1. 2. 'Sequent'

A sequent is a formal expression of the form

$$
\mathfrak{P}_{1}, \mathfrak{P}_{2}, \cdots \cdots, \mathfrak{P}_{m} \rightarrow \mathfrak{B}_{1}, \mathfrak{B}_{2}, \cdots \cdots, \mathfrak{B}_{n}
$$

where $m, n \geqq 0$ and $\mathfrak{A}_{1}, \mathfrak{A}_{2}, \ldots \ldots, \mathfrak{A}_{m}, \mathfrak{H}_{1}, \mathfrak{B}_{2}, \ldots \ldots, \mathfrak{B}_{n}$ are arbitrary formulae. The part $\mathfrak{A}_{1}, \mathfrak{P}_{2}, \ldots \ldots, \mathfrak{A}_{m}$ is called the antecedent, and $\mathfrak{B}_{1}, \mathfrak{B}_{2}, \ldots \ldots, \mathfrak{B}_{n}$ the succedent of the sequent.

We shall use Greek capitals, such as $\Gamma, \Delta, \Theta, \Lambda$ and so on, to represent finite sequences of zero or more formulae with separating formal commas included, when we wish to indicate sequences of formulae in antecedent or succedent without naming the formulae individualy.

1. 3. 'Rules of inference'

As rules of inference we use ones obtained from those for Gentzen's 'Kalkül $L K$ ', which are represented as the 'SchluBfiguren-Schemata', by adjoining the following

Rule of infercnce for mathematical induction:

$$
\frac{\mathfrak{F}(\mathfrak{a}), \Gamma \rightarrow \Theta, \mathfrak{F}\left(\mathfrak{a}^{\prime}\right)}{\mathfrak{F}(0), \Gamma \rightarrow \Theta, \mathfrak{F}(\mathrm{t})},
$$

where $\mathfrak{F}(\mathfrak{a})$ is an arbitrary formula, $\mathrm{t}$ is an arbitrary term, $\Gamma$ and $\Theta$ are arbitrary finite sequences of formulae, $\mathfrak{a}$ is an arbitrary free variable not contained in $\Gamma$ and $\Theta$, and $\mathfrak{F}(0), \mathfrak{F}(t)$ and $\mathfrak{F}\left(\mathfrak{a}^{\prime}\right)$ are the results of substituting $0, t$ and $\mathfrak{a}^{\prime}$ for $\mathfrak{a}$ throughout $\mathfrak{F}(\mathfrak{a})$, respectively.

The $L K$-inferences are divided into the structural and the logical inferences. The logical inferences constitute introductions of a logical symbol, but sometimes in the antecedent, and sometimes in the succedent. We shall denote, for example, an inference for the 'introduction of $V$ in 
succedent' briefly by ' $V$-succedent'. The logical inferences for the introductions of $7, \&, \vee, \supset$ are called the propositional inferences, and of $\forall$ and $B$ the predicate inferences.

1. 4. 'Proof'

As the (formal) proof, we use only the Gentzen's 'stammbaumförmige Herleitung' which has one lowermost sequent-the endsequent-and some uppermost sequents of the form

$$
\mathfrak{I} \rightarrow \mathfrak{I}
$$

where $\mathfrak{I}$ is an arbitrary formula.

A proof of a sequent is a proof which has the sequent as the endsequent. A sequent is said to be provable, if there exists a proof of the sequent.

1. 5. 'Calculi $L K$ and $L J$ '

A proof containing no inference for mathematical induction is called an $L K$-proof. A sequent is said to be $L K$-provable, if there exists an $L K$ proof of the sequent.

A sequent not containing more than one formula in the succedent is called an $L J$-sequent. An $L K$-proof containing only $L J$-sequents is called an $L J$-proof. A sequent is said to be $L J$-provable, if there exists an $L J$ proof of the sequent, accordingly an $L J$-provable sequent is an $L J$ sequent.

Gentzen [1] proved two following theorems, simultaneously formulating the Calculi $L K$ and $L J$.

1. 51. NORMAL FORM THEOREM. For any $L K$ - or $L J$-provable sequent, there exists an $L K$-or LJ-proof of the sequent which contains no cut, respectively.

1. 52. EXTENDED NORMAL FORM THEOREM ON $L K$. For any $L K$-provable sequent containing only prenex formulae, there exists an LK-proof of the sequent which contains no cut and in which there is an occurrence of $a$ sequent $\Xi$ such that no quantifiers occur in $\mathbb{\Xi}$, and the part of the proof from $\mathfrak{S}$ to the endsequent consists solely of structural and predicate inferences.

1. 6. The result proved by Gentzen [2] can be stated as follows:

CONSISTENCY THEOREM. Let $\Gamma_{0}$ be a finite sequence which contains only prenex closed formulae containing no existential quantifiers. If the sequent 


$$
\Gamma_{0} \rightarrow
$$

is provable, then the sequent is LK-provable.

\section{Normal axiom system}

A finite sequence of closed formulae is called an axiom system, and each of the closed formulae is called an axiom of the axiom system. A formula $\mathfrak{Q}$ is said to be provable from an axiom system $\Gamma_{0}$, if the sequent

$$
\Gamma_{0} \rightarrow \mathfrak{I}
$$

is provable. An axiom system is said to be contradictory, if there exists a formula $\mathfrak{N}$ such that $\mathfrak{P}$ and $7 \mathfrak{A}$ are both provable from the axiom system. An axiom system $\Gamma_{0}$ is contradictory, if and only if the sequent

$$
\Gamma_{0} \rightarrow
$$

is provable. An axiom system is said to be consistent, if it is not contradictory.

By $\Gamma_{a}$ we denote the following axiom system:

$$
\begin{aligned}
& \forall x(x=x), \\
& \forall x \forall y(x=y \supset y=x), \\
& \forall x \forall y \forall z\{(x=y \& y=z) \sqsupset x=z\}, \\
& \forall x \forall y\left(x=y \supset x^{\prime}=y^{\prime}\right), \\
& \forall x>\left(x^{\prime}=0\right), \\
& \forall x \forall y\left(x^{\prime}=y^{\prime} \supset x=y\right) .
\end{aligned}
$$

By Gentzen's Consistency theorem and Extended normal form theorem on $L K$, it can be immediately proved that $\Gamma_{a}$ is consistent.

An axiom system $\Gamma_{0}$ is said to be normal, if the following conditions are fulfilled:

1) $\Gamma_{0}$ contains all of the axioms of $\Gamma_{a}$.

2) Each of the axioms of $\Gamma_{0}$, except the axioms of $\Gamma_{a}$, has the form

$$
\forall \xi \mathfrak{H}(\xi) \text { or } \quad \forall \xi(\mathbb{}(\xi) \supset \mathfrak{P}(\xi))
$$

where $\mathfrak{P}(\xi)$ contains no logical symbols and $₫(\xi)$ contains no logical symbols except \&. 


\section{Equality axiom}

Let $\mathfrak{q}$ be a predicate symbol. Then the formula of the form

$$
\forall \boldsymbol{\xi} \forall \boldsymbol{\eta}\{(\boldsymbol{\xi}=\boldsymbol{\eta} \& \mathfrak{P}(\xi)) \supset \mathfrak{P}(\boldsymbol{\eta})\}
$$

is called the equality axiom for $\mathfrak{P}$, where $\xi=\eta$ means

$$
\mathfrak{x}_{1}=\mathfrak{y}_{2} \& \mathfrak{x}_{2}=\mathfrak{y}_{2} \& \cdots \cdots \& \mathfrak{x}_{n}=\mathfrak{y}_{n},
$$

if $\xi$ and $\eta$ are sequences of $n$ bound variables $\mathfrak{x}_{1}, \mathfrak{x}_{2}, \cdots \cdots, \mathfrak{x}_{n}$, and $\mathfrak{y}_{1}, \mathfrak{y}_{2}$, $\cdots \cdots, \mathfrak{y}_{n}$, respectively.

THEOREM 1. Let $\Gamma_{0}$ be an axiom system which contains only prenex formulae containing no existential quantifiers and which contains all of the axioms of $\Gamma_{a}$, and $\mathfrak{P}$ be a predicate symbol. If $\Gamma_{0}$ is consistent, then the axiom system

$$
\forall \xi \forall \eta\{(\xi=\eta \& \mathfrak{P}(\xi)) \supset \mathfrak{W}(\eta)\}, \Gamma_{0}
$$

is consistent.

Proof. Let us assume that the latter axiom system is contradictory, i. e. the sequent

$$
\forall \xi \forall \eta\{(\xi=\eta \& \Re(\xi)) \supset \mathfrak{P}(\eta)\}, \Gamma_{0} \rightarrow
$$

is provable, then it is $L K$-provable (Consistency theorem), accordingly there exists an $L K$-proof $\boldsymbol{P}$ of it such that the following conditions are fulfilled (Extended normal form theorem on $L K$ ):

1) $\boldsymbol{P}$ contains no cut.

2) $\boldsymbol{P}$ contains no free variable.

3) In $\boldsymbol{P}$, there is an occurrence of a sequent $\mathbb{S}$ such that no quantifiers occur in $\mathbb{S}$, and the part of $\boldsymbol{P}$ from $\subseteq$ to the endsequent consists solely of structural and predicate inferences.

The succedent of $\Theta$ is empty, and the formulae contained in the antecedent of $\subseteq$ are all provable from $\Gamma_{0}$, then the sequent

$$
\Gamma_{0} \rightarrow
$$

is provable, i. e. $\Gamma_{0}$ is cotradictory, q. e. d.

\section{Recursive function}

In the present formal system each function of $n$ variables defined for 
a11 non-negative integers $(n=0,1,2, \cdots)$, except the successor function, is represented by a predicate $\mathfrak{P}(\xi, \mathfrak{l})$ of $n+1$ arguments, in which $\xi$ means $n$ independent variables and $\mathfrak{y}$ means the dependent variable of the function. But for the sake of formulating that function in the formal system, of course, it is necessary that a consistent axiom system from which the formulae

$$
\forall \xi \not \mathfrak{H} \mathfrak{P}(\xi, \mathfrak{l}) \quad \text { and } \quad \forall \xi \forall \mathfrak{y} \forall \mathfrak{z}\{(\mathfrak{P}(\xi, \mathfrak{y}) \& \mathfrak{P}(\xi, \mathfrak{z})) \supset y=z\}
$$

are both provable can be founded. The function defined in the above way is nothing but the function denoted by the expression $\iota \mathfrak{y} \mathfrak{B}(\xi, \mathfrak{l})$ in the usual symbolism.

Let $\mathfrak{P}$ be a predicate symbol of $n+1$ argument places. If there exists a consistent normal axiom system from which the formulae

$$
\forall \xi\{\mathfrak{y} \mathfrak{P}(\xi, \mathfrak{y}) \quad \text { and } \quad \forall \xi \forall \mathfrak{y} \forall \mathfrak{z}\{(\mathfrak{P}(\xi, \mathfrak{y}) \& \mathfrak{P}(\xi, \mathfrak{z})) \supset \mathfrak{y}=\mathfrak{z}\}
$$

are provable, then the function $\iota \mathfrak{y} \mathfrak{P}(\xi, \mathfrak{y})$ is called a recursive function of $n$ variables.

4. 1. THEOREM 2. Let $\Gamma_{0}$ be a consistent normal axiom system,

$$
\Gamma_{0} \rightarrow \forall \xi \operatorname{Bg} \mathfrak{F}(\xi, \mathfrak{y})
$$

and

$$
\Gamma_{0} \rightarrow \forall \xi \forall \mathfrak{y} \forall\{(\mathfrak{F}(\xi, \mathfrak{y}) \& \mathfrak{F}(\xi, \mathfrak{z})) \supset \mathfrak{l}=\mathfrak{z}\}
$$

be provable sequents, and $\mathfrak{F}$ be a predicate symbol not contained in $\Gamma_{0}$ and $\mathfrak{F}(\xi, \mathfrak{y})$. If $\mathfrak{F}(\xi, \mathfrak{y})$ contains no free variables nor logical symbols except \&, $\vee, \mathcal{F}$, then there exists a consistent normal axiom system $\Gamma_{1}$ such that $\Gamma_{1}$ contains all of the axioms of $\Gamma_{0}$ and the sequent

$$
\left.\Gamma_{1} \rightarrow \forall \xi \forall \mathfrak{V}(\mathfrak{P}(\xi, \mathfrak{l})) \supset \mathfrak{F}(\xi, \mathfrak{l})\right)
$$

is provable.

Proof. We can assume, without loss of generality, that $\Gamma_{0}$ contains all of the equality axioms for the predicate symbols contained in $\mathfrak{F}(\xi, 1)$ (Theorem 1), and that $\mathfrak{F}(\xi, \mathfrak{y})$ has the form

$$
\left.\exists \eta_{1} \mathfrak{c}_{1}\left(\xi, \mathfrak{y}, \eta_{1}\right) \vee \mathcal{H} \eta_{2} \mathfrak{C}_{2}\left(\xi, \mathfrak{y}, \eta_{2}\right) \vee \cdots \cdots \vee \mathcal{H} \eta_{n} \mathfrak{c}_{n}(\xi, \mathfrak{y}), \eta_{n}\right)
$$

where $\left.\mathfrak{c}_{1}\left(\xi, \mathfrak{l}, \eta_{2}\right), \mathfrak{\mho}_{2}(\xi, \mathfrak{l}), \eta_{2}\right), \cdots \cdots, \mathfrak{c}_{n}\left(\xi, \mathfrak{l}, \eta_{n}\right)$ contain no logical symbols except \&. Let $\Gamma_{1}$ be the axiom system obtained from $\Gamma_{0}$ by adjoining the axioms 
$\forall \xi \forall \mathfrak{l}) \forall \eta_{i}\left(\mathfrak{l}_{\imath}\left(\xi, \mathfrak{y}, \eta_{\mathfrak{i}}\right) \sqsupset \mathfrak{P}(\xi, \mathfrak{y})\right) \quad(i=1,2, \cdots \cdots, n)$

and

$\forall \xi \forall \mathfrak{y} \forall \mathfrak{j}\{(\mathfrak{P}(\xi, \mathfrak{y}) \& \mathfrak{P}(\boldsymbol{\xi}, \mathfrak{z})) \supset \mathfrak{y}=\mathfrak{z}\}$.

Then the axiom system $\Gamma_{1}$ is normal, the sequent

$$
\Gamma_{1} \rightarrow \forall \xi \forall \mathfrak{v}(\mathfrak{P}(\xi, \mathfrak{l}) \supset \subset \mathfrak{F}(\xi, \mathfrak{l}))
$$

is provable, and $\Gamma_{1}$ is consistent because the axiom system

$$
\forall \xi \forall \mathfrak{l}(\mathfrak{H}(\xi, \mathfrak{y}) \supset \subset \mathfrak{F}(\xi, \mathfrak{y})), \Gamma_{0}
$$

from which the formulae

$$
\forall \xi \forall \mathfrak{y} \forall \eta_{i}\left(\mathbb{C}_{i}\left(\xi, y, \eta_{\imath}\right) \sqsupset \mathfrak{P}(\xi, \mathfrak{y})\right) \quad(i=1,2, \cdots, n)
$$

and

$$
\forall \xi \forall \mathfrak{y} \forall \mathfrak{z}\{(\mathfrak{B}(\xi, \mathfrak{y}) \& \mathfrak{P}(\xi, \mathfrak{z})) \supset \mathfrak{y}=\mathfrak{z}\}
$$

are all provable is consistent, q. e. d.

From this theorem we can see that the function obtained by substituting recursive functions fór some arguments of a recursive function is recursive.

4. 2. THEOREM 3. Let $\Gamma_{0}$ be a consistent normal axiom system, $\mathfrak{P}_{1}$ and $\mathfrak{P}_{2}$ be predicate symbols, the formulae

$\forall \xi \mathbb{H} \mathfrak{y} \mathfrak{P}_{1}(\xi, \mathfrak{y})$,

$\left.\forall \xi \forall \mathfrak{y} \forall \mathfrak{z} \mid\left(\mathfrak{P}_{1}(\xi, \mathfrak{y}) \& \mathfrak{P}_{1}(\xi, \mathfrak{z})\right) \supset \mathfrak{y}=\mathfrak{z}\right\}$,

$\forall \xi \forall \mathfrak{r} \forall \mathfrak{y}\left\{\mathfrak{H} \mathfrak{P}_{2}(\xi, \mathfrak{x}, \mathfrak{y}, \mathfrak{u})\right.$,

$\forall \xi \forall \mathfrak{x} \forall \mathfrak{y} \forall \mathfrak{u} \forall \mathfrak{v}\left\{\left(\mathfrak{P}_{2}(\xi, \mathfrak{k}, \mathfrak{y}, \mathfrak{u}) \& \mathfrak{P}_{2}(\xi, \mathfrak{x}, \mathfrak{y}, \mathfrak{v})\right) \sqsupset \mathfrak{u}=\mathfrak{v}\right\}$

be provable from $\Gamma_{0}, \mathfrak{P}$ be a predicate symbol which is distinct from $\mathfrak{\beta}_{1}, \mathfrak{P}_{2}$ and is not contained in $\Gamma_{0}$, and $\Gamma_{1}$ be the normal axiom system obtained from $\Gamma_{0}$ by adjoining the axioms

$\forall \xi \forall \mathfrak{h}\left(\mathfrak{P}_{1}(\xi, \mathfrak{y}) \supset \mathfrak{P}(\xi, 0, \mathfrak{y})\right)$,

$\forall \xi \forall \mathfrak{x} \forall \mathfrak{y} \forall \mathfrak{u}\left\{\left(\mathfrak{P}(\xi, \mathfrak{x}, \mathfrak{y}) \& \mathfrak{P}_{2}(\boldsymbol{\xi}, \mathfrak{x}, \mathfrak{y}, \mathfrak{u})\right) \supset \mathfrak{P}\left(\xi, \mathfrak{l}^{\prime}, \mathfrak{u}\right)\right\}$,

$\forall \xi \forall \mathfrak{y n} \forall \mathfrak{z}\{(\mathfrak{P}(\xi, \mathfrak{x}, \mathfrak{y}) \& \mathfrak{P}(\xi, \mathfrak{x}, \mathfrak{z}))=\mathfrak{y}=\mathfrak{z}\}$.

Then, $\Gamma_{1}$ is consistent and

$$
\Gamma_{1} \rightarrow \forall \xi \forall \mathfrak{g} \mathfrak{g} \mathfrak{P}(\xi, \mathfrak{x}, \mathfrak{y})
$$

is provable (Primitive recursion).

Proof. The formulae

$$
\forall \xi \exists \mathfrak{H} \mathfrak{P}(\xi, 0, \mathfrak{y})
$$


and

$$
\forall \mathfrak{x}\left(\forall \xi \exists \mathfrak{H} \mathfrak{P}(\boldsymbol{\xi}, \mathfrak{x}, \mathfrak{y}) \supset \forall \xi \xi \mathfrak{y} \mathfrak{P}\left(\boldsymbol{\xi}, \mathfrak{x}^{\prime}, \mathfrak{y}\right)\right)
$$

are both provable from $\Gamma_{1}$. Hence

$$
\forall \boldsymbol{\xi} \forall \mathfrak{x} \mathfrak{g} \mathfrak{\mathfrak { P }}(\boldsymbol{\xi}, \mathfrak{x}, \mathfrak{y})
$$

is provable from $\Gamma_{1}$.

In the following we assume, without loss of generality, that $\Gamma_{0}$ contains the equality axiom for $\mathfrak{\beta}_{2}$ (Theorem 1 ).

Let $\Gamma_{1}$ be contradictory, i. e. the sequent

$$
\Gamma_{1} \rightarrow
$$

be provable, then the sequent is $L K$-provable (Consistency theorem), accordingly there exists an $L K$-proof $\boldsymbol{P}$ such that the following conditions are fulfilled (Extended normal form theorem on $L K$ ):

1) The endsequent of $\boldsymbol{P}$ is $\Gamma_{1} \rightarrow$.

2) $\boldsymbol{P}$ contains no cut.

3) $\boldsymbol{P}$ contains no free variable.

4) In $\boldsymbol{P}$, there is an occurrence of a sequent $\mathbb{S}$ such that no quantifiers occur in $\mathfrak{S}$, and the part of $\boldsymbol{P}$ from $\mathfrak{S}$ to the endsequent consists solely of structural and predicate inferences.

The succedent of $\mathfrak{S}$ is empty.

For any formula $\mathfrak{A}$ containing no quantifiers, we define a formula denoted by $\mathfrak{A}^{*}$, recursively, as follows :

1) If $\mathfrak{A}$ is a predicate symbol distinct from $\mathfrak{P}$ and $\boldsymbol{\tau}$ is a sequence of terms, $(\mathfrak{D}(\boldsymbol{\tau}))^{*}$ is $\mathfrak{D}(\boldsymbol{\tau})$.

2) If $t$ is a term and $\tau$ is a sequence of $\operatorname{terms,}(\mathfrak{P}(\tau, 0, t))^{*}$ is $\mathfrak{P}_{1}(\tau, \mathfrak{t})$.

3) If $\mathfrak{a}$ is a free variable, $t$ is a term and $\tau$ is a sequence of terms, $(\mathfrak{P}(\boldsymbol{\tau}, \mathfrak{a}, \mathfrak{t}))^{*}$ is $\mathfrak{P}(\boldsymbol{\tau}, \mathfrak{a}, \mathfrak{t})$.

4) If $\mathfrak{s}$ and $t$ are terms and $\tau$ is a sequence of terms, $\left(\mathfrak{P}\left(\tau, \mathfrak{s}^{\prime}, \mathfrak{t}\right)\right)^{*}$ is $\mathbb{F} \mathfrak{l}\left((\mathfrak{P}(\boldsymbol{\tau}, \mathfrak{s}, \mathfrak{y})) * \& \mathfrak{P}_{2}(\boldsymbol{\tau}, \mathfrak{s}, \mathfrak{y}, \mathfrak{t})\right)$.

5) If $\mathfrak{P}$ is a formula, $\left(\boldsymbol{Z M}^{*}\right)^{*}$ is $\boldsymbol{\supset \mathfrak { H }}^{*}$.

6) If $\mathfrak{N}$ and $\mathfrak{B}$ are formulae, $(\mathfrak{A} \& \mathfrak{P})^{*},(\mathfrak{A} \vee \mathfrak{B})^{*}$ and $(\mathfrak{A} \supset \mathfrak{B})^{*}$ are $\mathfrak{U}^{*} \&$ $\mathfrak{B}^{*}, \mathfrak{U}^{*} \vee \mathfrak{B}^{*}$ and $\mathfrak{U}^{*} \supset \mathfrak{B}^{*}$, respectively.

Now, let $\mathfrak{S}$ be 


$$
\mathfrak{A}_{1}, \mathfrak{A}_{2}, \cdots \cdots, \mathfrak{A}_{n} \rightarrow
$$

Then

$$
\mathfrak{A}^{*}, \mathfrak{A}^{*}, \cdots \cdots, \mathfrak{A}^{*}{ }_{n} \rightarrow
$$

is provable. On the other hand, the formulae $\mathfrak{A}^{*}, \mathfrak{U}^{*}{ }_{2}, \cdots \cdots, \mathfrak{U}^{*}{ }_{n}$ are alI provable from $\Gamma_{0}$. Hence

$$
\Gamma_{0} \rightarrow
$$

is provable, i. e. $\Gamma_{0}$ is contradictory, q. e. d.

4. 3. THEOREM 4. Let $\Gamma_{0}$ be a consistent normal axiom system which contains the axioms

$\forall x \forall u(u=x \supset \mathrm{S}(x, 0, u))$,

$\forall x \forall y \forall u \forall v\left\{\left(\mathrm{~S}(x, y, u) \& v=u^{\prime}\right) \supset \mathrm{S}\left(x, y^{\prime}, v\right)\right\}$,

$\forall x \forall y \forall u \forall v\{(\mathrm{~S}(x, y, v) \& \mathrm{~S}(x, y, v)) \sqsupset u=v\}$

and from $\Gamma_{0}$ the formulae

$\forall \xi \forall \mathfrak{y} \mathbb{u} \mathfrak{u} \mathfrak{B}_{0}(\xi, \mathfrak{y}, \mathfrak{u})$,

$\forall \xi \forall \mathfrak{y} \forall \mathfrak{u} \forall \mathfrak{v}\left\{\left(\mathfrak{P}_{0}(\xi, \mathfrak{y}, \mathfrak{u}) \& \mathfrak{P}_{0}(\xi, \mathfrak{y}, \mathfrak{v})\right) \supset \mathfrak{u}=\mathfrak{v}\right\}$,

$\forall \xi \mathfrak{g} \mathfrak{M} \mathfrak{P}_{0}(\xi, \mathfrak{y}, 0)$

be provable, where $\mathfrak{P}_{0}$ is a predicate symbol, and $\mathfrak{P}$ be a predicate symbol which is distinct from $\mathfrak{P}_{0}$ and is not contained in $\Gamma_{0}$.

Then there exists an another consistent normal axiom system which contains all of the axioms of $\Gamma_{0}$, and from which the formulae

$\forall \boldsymbol{\xi} \mathbb{g} \mathfrak{y}(\xi, \mathfrak{y})$,

$\forall \boldsymbol{\xi} \forall \mathfrak{y} \forall \mathfrak{z}\{(\mathfrak{P}(\xi, \mathfrak{y}) \& \mathfrak{P}(\xi, \mathfrak{z})) \supset \mathfrak{y}=\mathfrak{z}\}$,

$\forall \boldsymbol{V} \forall \mathfrak{y}\left(\mathfrak{P}(\xi, \mathfrak{y}) \supset \mathfrak{P}_{0}(\xi, \mathfrak{y}, 0)\right)$,

$\forall \boldsymbol{\xi} \forall \mathfrak{y} \forall \mathfrak{z}\left\{\left(\mathfrak{P}(\boldsymbol{\xi}, \mathfrak{y}) \& \mathfrak{P}_{0}(\boldsymbol{\xi}, \mathfrak{z}, 0)\right) \supset \mathfrak{y} \leqq z\right\}$

are provable, where $\mathfrak{y} \leqq \mathfrak{z}$ means $\mathbb{B} x \mathbf{S}(x, \mathfrak{y}, \mathfrak{z})$.

The function $\iota \mathfrak{y} \mathfrak{P}(\xi, \mathfrak{y})$ defind in this theorem is nothing but the function denoted by the expression $\mu \mathfrak{y} \mathfrak{P}_{0}(\xi, \mathfrak{y}, 0)$ which means the least $\mathfrak{y}$ such that $\mathfrak{P}_{0}(\xi, \mathfrak{y}, 0)$.

Proof. We can assume, without loss of generality, that $\Gamma_{0}$ contains the following axioms (Theorem 3):

$\forall x \forall u(u=0 \supset \mathrm{P}(x, 0, u))$,

$\forall x \forall y \forall u \forall v\left\{(\mathrm{P}(x, y, u) \& \mathrm{~S}(u, x, v)) \supset \mathrm{P}\left(x, y^{\prime}, v\right)\right\}$, 
$\forall x \forall y \forall u \forall v\{(\mathrm{P}(x, y, u) \& \mathrm{P}(x, y, v)) \supset u=v\}$,

$\forall y(y=0 \supset \operatorname{Sg}(0, y))$,

$\forall x \forall y \forall z\left\{(\operatorname{Sg}(x, y) \& z=1) \supset \mathrm{Sg}\left(x^{\prime}, z\right)\right\}$,

$\forall x \forall y \forall z\{(\operatorname{Sg}(x, y) \& \operatorname{Sg}(x, z)) \supset y=z\}$.

Let $\mathfrak{f}(\xi, \mathfrak{y}, \mathfrak{z}, \mathfrak{u})$ mean $\mathbb{H} \mathfrak{w}\left(\mathfrak{P}_{0}(\xi, \mathfrak{y}, \mathfrak{w})\right.$ \& $\left.\mathrm{P}(\mathfrak{z}, \mathfrak{w}, \mathfrak{b})\right)$, then the formulae $\forall \boldsymbol{\xi} \forall \mathfrak{y} \forall \mathfrak{z} \mathbb{Z} \mathfrak{u} \mathfrak{F}(\xi, \mathfrak{y}, \mathfrak{z}, \mathfrak{u})$

and

$\forall \xi \forall \mathfrak{y} \forall \mathfrak{z} \forall \mathfrak{u} \forall \mathfrak{b}\{(\mathfrak{F}(\xi, \mathfrak{y}, \mathfrak{\jmath}, \mathfrak{u}) \quad \& \quad \mathfrak{F}(\xi, \mathfrak{y}, \mathfrak{z}, \mathfrak{b})) \supset \mathfrak{u}=\mathfrak{v}\}$

are provable from $\Gamma_{0}$. Hence, there exists a consistent normal axiom system $\Gamma_{1}$ which contains all of the axioms of $\Gamma_{0}$ and from which the formula

$\forall \xi \forall \mathfrak{y} \forall \mathfrak{z} \mathfrak{u}\left(\mathfrak{P}_{1}(\xi, \mathfrak{y}, \mathfrak{z}, \mathfrak{u}) \supset \subset \mathfrak{F}(\xi, \mathfrak{y}, \mathfrak{z}, \mathfrak{u})\right)$

is provable, where $\mathfrak{P}_{1}$ is a predicate symbol distinct from $\mathfrak{P}, \mathfrak{P}_{0}$ and not contained in $\Gamma_{0}$ (Theorem 2).

Let $\Gamma_{2}$ be the normal axiom system obtained from $\Gamma_{1}$ by adjoining the following axioms:

$\forall \xi, \forall \mathfrak{u}\left(\mathfrak{u}=1 \supset \mathfrak{P}_{2}(\xi, 0, \mathfrak{u})\right)$,

$\forall \xi \forall \mathfrak{y} \forall \mathfrak{z} \forall \mathfrak{u}\left\{\left(\mathfrak{P}_{2}(\xi, \mathfrak{y}, \mathfrak{z}) \& \mathfrak{P}_{1}(\xi, \mathfrak{y}, \mathfrak{z}, \mathfrak{u})\right) \supset \mathfrak{P}_{2}\left(\xi, \mathfrak{y}^{\prime}, \mathfrak{u}\right)\right\}$,

$\forall \mathfrak{\xi} \forall \mathfrak{y} \forall \mathfrak{u} \forall \mathfrak{v}\left\{\left(\mathfrak{B}_{2}(\xi, \mathfrak{y}, \mathfrak{u}) \& \mathfrak{P}_{2}(\xi, \mathfrak{y}, \mathfrak{v})\right) \supset \mathfrak{u}=\mathfrak{v}\right\}$,

where $\mathfrak{P}_{2}$ is a predicate symbol distinct from $\mathfrak{P}, \mathfrak{P}_{0}, \mathfrak{P}_{1}$ and not contained in $\Gamma_{1}$. Then $\Gamma_{2}$ is consistent (Theorem 3).

Now, let $\mathfrak{B}(\xi, \mathfrak{y})$ mean

$$
\mathbb{Z} \mathfrak{u}\left(\operatorname{Sg}(\mathfrak{u}, 1) \& \mathfrak{P}_{2}(\xi, \mathfrak{y}, \mathfrak{u}) \& \mathfrak{P}_{2}\left(\xi, \mathfrak{y}^{\prime}, 0\right)\right),
$$

then

$\forall \boldsymbol{\xi} \mathbb{B} \mathfrak{y}(\xi) \mathfrak{y})$,

$\forall \xi \forall \mathfrak{y} \forall \mathfrak{z}\{(\mathbb{S}(\xi, \mathfrak{y}) \& \mathbb{B}(\xi, \mathfrak{z})) \supset \mathfrak{y}=\mathfrak{z}\}$,

$\forall \xi \forall \mathfrak{y}\left(\mathbb{S}(\xi, \mathfrak{y}) \supset \mathfrak{P}_{0}(\xi, \mathfrak{y}, 0)\right)$,

$\forall \xi \forall \mathfrak{y} \forall \mathfrak{z}\left\{\left(\mathcal{G}(\xi, \mathfrak{y}) \& \mathfrak{P}_{0}(\xi, \mathfrak{z}, 0)\right) \sqsupset \mathfrak{y} \leqq \mathfrak{z}\right\}$

are all provable from $\Gamma_{2}$. Hence, by Theorem 2, it is almost clear that there exists a consistent normal axiom system required in the Theorem, q. e. d. 


\section{Calculability of recursive function's}

In the Proof of Theorem 5, we shall use the following Lemmas 1 and 2 , the former of which is the immediate consequence from the result of $\S 1$ Maehara [3], and the latter can be simply proved by Gentzen's Normal form theorem on $L J$.

LEMMA 1. If the endsequent of an LK-proof containing no $フ-, コ-$ nor $V$-succedent is an $L J$-sequent, then the endsequent is $L J$-provable.

LEMMA 2. Let $\Gamma_{0}$ be an axiom system which contains only prenex formulae containing no logical symbol $\vee$ nor $\mathbb{Z}$, and $\mathbb{Z} \mathfrak{Y} \mathfrak{F}(\mathfrak{y})$ be a closed formula of such form. If the sequent

$$
\Gamma_{0} \rightarrow \mathfrak{B} \mathfrak{y} \mathfrak{F}(\mathfrak{y})
$$

is $L J$-provable, then there exists a numeral $\mathfrak{n}$ such that the sequent

$$
\Gamma_{0} \rightarrow \mathfrak{F}(\mathfrak{n})
$$

is $L J$-provable.

THEOREM 5. Let $\Gamma_{0}$ be a consistent normal axiom system, $\mathfrak{P}$ be a predicate symbol, and the formulae

$$
\forall \xi \mathfrak{g} \mathfrak{y} \mathfrak{P}(\xi, \mathfrak{y})
$$

and

$$
\forall \xi \forall \mathfrak{y} \forall \mathfrak{z}\{(\mathfrak{P}(\xi, \mathfrak{y}) \& \mathfrak{P}(\boldsymbol{\xi}, \mathfrak{z})) \supset \mathfrak{y}=\mathfrak{z}\}
$$

be provable from $\Gamma_{0}$. If $\nu$ is a sequence of arbitrary numerals, then there exists only one numeral $\mathfrak{n}$ such that the sequent

$$
\Gamma_{0} \rightarrow \mathfrak{P}(\boldsymbol{\nu}, \mathfrak{n})
$$

is $L J$-provable.

PRoor. Let $\nu$ be a sequence of numerals, then the sequent

$$
\Gamma_{0} \rightarrow \mathbb{Z} \mathfrak{y} \mathfrak{P}(\nu, \mathfrak{y})
$$

is provable, accordingly the sequent

$$
\forall \mathfrak{y}>\mathfrak{P}(\nu, \mathfrak{y}), \Gamma_{0} \rightarrow
$$

is provable. By Gentzen's Consistency theorem, the latter sequent is $L K$ provable, hence so is the former, accordingly there exists an $L K$-proof of the former sequent which contains no cut (Normal form theorem). Then, that $L K$-proof contains no 7 -, $コ$ - nor $V$-succedent, accordingly the 
endsequent is $L J$-provable (Lemma 1 ). Hence, there exists a numeral $\mathfrak{n}$ such that the sequent

$$
\Gamma_{0} \rightarrow \mathfrak{P}(\nu, \mathfrak{n})
$$

is $L J$-provable, because each of the axioms of $\Gamma_{0}$ contains no logical symbol $V$ nor $H$ (Lemma 2).

The uniqueness of such a numeral $\mathfrak{n}$, from the consistency of $\Gamma_{0}$, is almost clear, q. e. d.

\section{References}

[1] G. Gentzen, Untersuchungen über das logische Schließen. Math. Zeitschr., 39, 176-210, 405-431 (1935).

[2] G. Gentzen, Neue Fassung des" Widerspruchsfreiheitsbeweises für die reine Zahlentheorie. Forschungen zur Logik und zur Grundlegung der exakten Wissenschaften, Neue Folge 4, 19-44 (1938).

[3] S. Maehara, Eine Darstellung der intuitionistischen Logik in der klassischen. Nagoya Math. Journ., 7, 45-64 (1954). 